\title{
Common carotid artery stiffness in patients with Spontaneous Coronary Artery Dissection (SCAD) is comparable to non-dissection controls
}

*Fahad F Almutairi ( $\nabla$ fahad.fa15@hotmail.com )

University of Leicester https://orcid.org/0000-0002-4509-7753

*Abtehal Al-hussaini

University of Leicester Department of Cardiovascular Sciences

Anne-Marie Marsh

University of Leicester Department of Cardiovascular Sciences

Nilesh J Samani

University of Leicester Department of Cardiovascular Sciences

\section{Gerry P McCann}

University of Leicester Department of Cardiovascular Sciences

David Adlam

Tokyo Daigaku Daigakuin Yakugakukei Kenkyuka Yakugakubu

Emma Chung

University of Leicester Department of Cardiovascular Sciences

Kumar V Ramnarine

University of Leicester Department of Cardiovascular Sciences

\section{Research}

Keywords: Ultrasound, carotid artery, Young's modulus, stiffness, shear wave elastography, spontaneous coronary artery dissection

Posted Date: May 22nd, 2020

DOI: https://doi.org/10.21203/rs.3.rs-30725/v1

License: (c) (i) This work is licensed under a Creative Commons Attribution 4.0 International License. Read Full License 


\section{Abstract}

Background: Arterial dissection is a rare but potentially life-threatening condition, hypothesized to be linked to changes in vessel wall elasticity. This study aimed to provide an insight into the pathophysiology of arterial dissection by assessing whether elasticity of the common carotid artery (CCA) differs between patients with confirmed spontaneous coronary artery dissection (SCAD) and nondissection controls.

Methods: Ultrasound shear wave elastography (SWE) estimates of artery wall elasticity were obtained from the left and right CCAs of 89 confirmed SCAD patients and 38 non-dissection controls. SWE images obtained over multiple cardiac cycles were analysed by a blinded observer to estimate elasticity in the form of a Young's Modulus (YM) value, across regions of interest (ROI) located within the anterior and posterior CCA walls. A confidence limit on the difference in mean YM between SCAD patients and nondissection controls was estimated.

Results: YM estimates ranged from 17 to $133 \mathrm{kPa}$ in SCAD patients compared to 34 to $87 \mathrm{kPa}$ in nondissection controls. The mean YM of 55 [SD: 21] kPa in SCAD patients was not significantly different to the mean of 57 [SD: 12] kPa in controls, $p=0.32$. The difference between groups was $2 \mathrm{kPa}$ [95\% $\mathrm{Cl}:-11$, 4].

Conclusions: We found no evidence of a significant difference in elasticity of the CCA between SCAD patients and non-dissection controls. This suggests that widespread changes in arterial compliance are not a major factor in the pathogenesis of arterial dissection.

\section{Background}

Arterial dissection of the major arteries is caused by the development of a false lumen within the tunica media of the artery. Clinical sequelae depend on the site of dissection, but can include myocardial infarction (for SCAD) [1], and stroke, in the event of dissection of the carotid or vertebral (cervical) arteries.

The underlying causes of arterial dissection are not well understood, but have been hypothesized to be linked to changes in vessel wall biomechanics. Dissection events often affect young to middle-aged people with few risk factors for atherosclerotic cardiovascular disease [2, 3]. In SCAD, pregnancy, in particular, appears to place otherwise healthy women at increased risk of dissection, especially in the late third trimester and early post-partum period [4]. Identification of non-invasive imaging biomarkers for identification of patients at risk of dissection would be highly beneficial.

Although the pathogenesis of dissection is currently unclear, SCAD has been demonstrated to be associated with remote arteriopathies, such as fibromuscular dysplasia (FMD) in the renal and carotid arteries $[5,6]$. Previous research suggests that increased arterial stiffness might make arteries more vulnerable to dissection [7], with more elastic vessels prone to aneurysms. Calvet et al. previously 
examined arterial wall motion over the cardiac cycle to estimate Young's Modulus (YM) in a small sample of 32 SCAD patients and found abnormal elastic properties of the carotid artery in patients with dissection [8].

This study adopts the newer technique of ultrasound shear wave elastography (SWE) to directly quantify CCA stiffness in SCAD patients compared to non-dissection controls. SWE imparts focused ultrasound 'push pulses' within tissue, generating a propagating acoustic 'shear wave'. The velocities of the resultant shear waves are measured and analysed by ultrafast ultrasound imaging to display a real-time 2D (elastogram) map of tissue stiffness [9]. The feasibility of quantifying YM in vessels has been established through in vitro and ex vivo studies $[10,11]$. Moreover, recent clinical studies investigating vascular applications of SWE have shown promising results highlighting the potential clinical value of SWE in vessel wall and plaque characterisation [12-14].

This study assessed carotid arterial wall stiffness in patients with confirmed SCAD for blinded comparison with non-dissection controls. The aim of the study was to provide a better understanding of SCAD pathophysiology, and to assess whether YM estimation might be useful as a diagnostic biomarker relating to the risk of arterial dissection.

\section{Methods}

\section{Participants and study protocol}

This study was sponsored by the University Hospitals of Leicester NHS Trust under NHS Research Ethics Committee Health Research Authority approval (REC reference 14/EM/0056). All participants provided written, fully informed, consent.

Included patients had angiographically confirmed SCAD diagnosed by at least two experienced clinicians. As part of the study protocol, body mass index (BMI), and blood pressure (BP) were measured. Demographic information (age and sex) and risk factors for cardiovascular disease (hypertension, hypercholesterolemia, diabetes mellitus, and family history of cardiovascular disease) were also recorded.

Ultrasound scans of the left and right common carotid arteries (CCA) were performed in SCAD patients and non-dissection controls using a SWE ultrasound scanner equipped with a $15-4 \mathrm{MHz}$ linear array probe (Axiplorer, Supersonic Imagine, France). The following SWE scanner settings were selected to optimise image quality: maximum acoustic power output, mid-range smoothing (6), persistence off, penetration mode, and colour map range up to $300 \mathrm{kPa}$. Approximately 10 seconds of cine-loop visualising longitudinal sections of the artery located $2 \mathrm{~cm}$ proximal to the carotid bulb were acquired. $\mathrm{A}$ typical cine-loop used for analysis is included as a supplemental movie file.

SWE scans were analysed retrospectively by an experienced observer, who was blinded to whether data came from a SCAD subject or non-dissection control. Arterial wall stiffness was quantified using the 
Aixplorer's built-in analysis software to measure mean Young's modulus from several $2 \mathrm{~mm}$ Regions of Interest (ROIs). From the acquired cine-loops, the first two SWE frames were discarded to enable the SWE acquisition to settle. Five consecutive frames were then analysed to estimate mean YM within each ROI. Two ROIs were placed on the anterior wall and two on the posterior wall of the left and right carotid arteries, as shown in Fig. 1. This resulted in a total of 40 YM measurements per participant ( 4 ROIs * 5 frames * 2 sides). Criteria for inclusion of measurements in further statistical analyses were: (1) good image quality; (2) complete filling of the shear wave elastogram; (3) adequate cine-loops (over a minimum of five frames). Exclusion criteria included: (1) poor image quality; (2) insufficient shear wave elastogram filling; (3) cine-loops with fewer than five SWE frames. Recordings were assessed for quality by an independent assessor who was blinded to whether images originated from patients or controls.

\section{Statistical analysis}

Data were analysed using GRAPHPAD PRISM ${ }^{\circledR}$ version 7 software (Prism, California, USA). Underlying statistical assumptions regarding normality were tested using a Shapiro-Wilks test. Demographic factors for SCAD patients and non-dissection controls were compared using Student's t-test for normally distributed data, and non-parametric tests (Wilcoxon and Mann-Whitney tests) for non-normally distributed variables. As YM estimates were normally distributed, an independent samples Student's t-test was used to compare average elasticity estimates for SCAD compared to those of non-dissection controls, and to estimate $95 \%$ confidence limits on the mean difference between groups. A p-value of $p<$ 0.05 was considered statistically significant.

\section{Results}

\section{Demographic and clinical characteristics}

A total of 127 carotid examinations from 38 non-dissection controls ( 35 females: 3 males) and 89 SCAD patients (87 females: 2 males) were performed. The demographic and clinical characteristics of SCAD and non-dissection control groups are summarised and compared in Table 1. Significant differences $(p<$ 0.0001) were observed for age and blood pressure parameters. On average, SCAD patients were 5 years older than the control group (SCAD: median age 48 years [range: 43 to 53 years]; controls: median age 43 years [range 36 to 49 years], $p<0.001)$. Systolic and diastolic pressures were both significantly lower in the SCAD group, with a mean (SD) systolic pressure in SCAD patients of 119 (16) $\mathrm{mmHg}$ compared to 130 (18) $\mathrm{mmHg}$ in non-dissection controls $(p=0.001)$; the mean (SD) diastolic pressure of $72(12) \mathrm{mmHg}$ in SCAD patients was also significantly lower than the $78(11) \mathrm{mmHg}$ in non-dissection controls $(p<$ 0.009 ), see Table 1. 
Table 1

Comparison of demographic characteristics and YM estimates for SCAD patients versus nondissection controls.

\begin{tabular}{|c|c|c|c|}
\hline $\operatorname{SCAD}(n=89)$ & Controls $(n=38)$ & Difference & \\
\hline Sex, female: male & $87: 2$ & $35: 3$ & $p=0.32^{1}$ \\
\hline Age, years (range) & $48(43-53)$ & $43(36-49)$ & $p<0.001^{3}$ \\
\hline $\mathrm{BMI}, \mathrm{kg} / \mathrm{m}^{2}$ (range) & $25(22-29)$ & $25(21-29)$ & $p=0.99^{3}$ \\
\hline Diastole, mmHg (SD) & $72(12)$ & $78(11)$ & 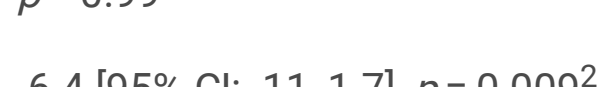 \\
\hline Systole, mmHg (SD) & $119(16)$ & $130(18)$ & $-0.4[95 \%(1 .-11,-1.1], p=0.009$ \\
\hline Hypertension, $n(\%)$ & $18(20 \%)$ & $\mathrm{N} / \mathrm{A}$ & $11[95 \% \mathrm{Cl}:-18,-4.6], p=0.001^{2}$ \\
\hline Hypercholesterolemia, $n(\%)$ & $7(8 \%)$ & N/A & - \\
\hline Diabetes Mellitus, $n(\%)$ & $7(8 \%)$ & $\mathrm{N} / \mathrm{A}$ & - \\
\hline Family History, $n$ (\%) & $56(63 \%)$ & $\mathrm{N} / \mathrm{A}$ & - \\
\hline \multirow[t]{2}{*}{ Young's Modulus, kPa (SD) } & $55(21)$ & $57(12)$ & - \\
\hline & & & $2[95 \% \mathrm{Cl}:-11,4], p=0.32^{2}$ \\
\hline
\end{tabular}

\section{CCA mean YM in SCAD patients compared to non- dissection controls}

Measurements from all Rols; left and right carotid arteries, anterior and posterior walls, across 5 frames for each subject, were averaged to provide a single estimate of the mean YM for each individual participant. Mean YM estimates were highly variable, ranging from 17 to $133 \mathrm{kPa}$ in SCAD patients and between 34 and $87 \mathrm{kPa}$ in controls. CCA YM estimates were confirmed to be normally distributed, with a mean (SD) YM of 55 (21) $\mathrm{kPa}$ in SCAD patients compared to 57 (12) $\mathrm{kPa}$ in non-dissection controls (Fig. 2). An unpaired (independent samples) Student's t-test confirmed no significant difference in mean YM between SCAD and control subjects $(p=0.32)$. The difference between groups was estimated to be $2 \mathrm{kPa}[95 \% \mathrm{Cl}:-11,4]$ suggesting that any difference between SCAD patients and non-dissection controls, if one exists, must be less than $11 \mathrm{kPa}$.

\section{Discussion}

In this study, we perform a first SWE comparison of YM estimates of common carotid artery stiffness in SCAD patients compared to non-dissection controls, with the aim of providing a better understanding of the pathophysiology of SCAD. The mean YM of the CCA in patients with confirmed SCAD was estimated to be within 2 [95\% Cl: -11 to 4] $\mathrm{kPa}$ of non-dissection controls, with no significant difference between groups, $p=0.34$. This result is at variance with a previous smaller study by Calvet et al., that identified a 
significantly higher CCA stiffness in patients with spontaneous cervical artery dissection [8]. Calvet et al. estimated YM through analysis of vessel wall compliance using ultrasound echo-tracking, and concluded that higher stiffness of the carotid wall and higher circumferential wall stress increases the risk of dissection. In this larger study, we directly measuring YM using SWE and found very similar YM between groups and no significant difference in YM between SCAD patients and controls. Secondly, we found that SWE measurements generate a wide range of YM estimates making practical utility of this approach in clinical practice limited. Adjusting for the power of our study, the $95 \%$ confidence limits of -11 to $+4 \mathrm{kPa}$ for the difference between groups is narrow compared to measurement variability, and includes zero signalling there may be no difference.

SWE was initially developed for assessing bulk homogeneous tissues, such as the liver, therefore, several challenges and limitations are associated with measurement of YM in arteries. The relationship between shear wave velocity and YM is based on theoretical assumptions of constant density, homogeneity, isotropy, and incompressibility, which may not be valid in vessels. In particular, slender vessels support Lamb wave propagation requiring a different theoretical model for YM estimation $[9,14,15]$. Our study is limited by the high variability observed in SWE CCA YM measurements. Further work would be beneficial for understanding sources of YM variability to determine whether these reflect true heterogeneity in tissue stiffness, or are a result of limitations of SWE when applied to vessels.

\section{Conclusions}

This study found no significant difference in mean YM of the CCA between SCAD patients and nondissection controls. The controls were slightly younger and with higher blood pressure than the SCAD group, but this is unlikely to have influenced our findings. We conclude that major artery stiffening is not a significant factor in the pathogenesis of arterial dissection in SCAD patients. This suggests that widespread changes in arterial compliance are not a major factor in the pathogenesis of dissection.

\section{Declarations}

- Ethics approval and consent to participate:

(REC reference 14/EM/0056).

- Consent for publication:

Not applicable

- Availability of data and materials:

The datasets used and/or analysed during the current study are available from the corresponding author on reasonable request.

- Competing interests: 
The authors declare that they have no competing interests.

- Funding acknowledgements:

This study was supported by the British Heart Foundation (BHF) PG/13/96/30608, the NIHR rare disease translational collaboration, the Leicester NIHR Biomedical Research Centre and BeatSCAD.

- Authors' contributions:

The study was conceived and designed by DA, NS, GM and KVR. AA and AM recruited and scanned patients. Data analysis was performed by FA, KVR and EC. Manuscript was drafted by FA, KVR and EC. All authors read, revised, and approved the manuscript.

\section{References}

1. Sharma, S., et al., Sudden cardiac death in patients with spontaneous coronary artery dissection. Journal of the American College of Cardiology, 2017. 70(1): p. 114-115.

2. Adlam, D., et al., European Society of Cardiology, acute cardiovascular care association, SCAD study group: a position paper on spontaneous coronary artery dissection. European heart journal, 2018.

3. Hayes, S.N., et al., Spontaneous coronary artery dissection: current state of the science: a scientific statement from the American Heart Association. Circulation, 2018. 137(19): p. e523-e557.

4. Saw, J., Pregnancy-associated spontaneous coronary artery dissection represents an exceptionally high-risk spontaneous coronary artery dissection cohort. 2017, Am Heart Assoc.

5. Saw, J., et al., Spontaneous coronary artery dissection: prevalence of predisposing conditions including fibromuscular dysplasia in a tertiary center cohort. JACC: Cardiovascular Interventions, 2013. 6(1): p. 44-52.

6. Saw, J., et al., Spontaneous coronary artery dissection: association with predisposing arteriopathies and precipitating stressors and cardiovascular outcomes. Circulation: Cardiovascular Interventions, 2014. 7(5): p. 645-655.

7. Callaghan, F.M., et al., Wall stress of the cervical carotid artery in patients with carotid dissection: a case-control study. American Journal of Physiology-Heart and Circulatory Physiology, 2011. 300(4): p. $\mathrm{H} 1451-\mathrm{H} 1458$.

8. Calvet, D., et al., Increased stiffness of the carotid wall material in patients with spontaneous cervical artery dissection. Stroke, 2004. 35(9): p. 2078-2082.

9. Couade, M., et al., Quantitative assessment of arterial wall biomechanical properties using shear wave imaging. Ultrasound in medicine \& biology, 2010. 36(10): p. 1662-1676.

10. Săftoiu, A., et al., The EFSUMB guidelines and recommendations for the clinical practice of elastography in non-hepatic applications: update 2018. Ultraschall in der Medizin-European Journal of Ultrasound, 2019. 40 (04), 425-453. 
11. Widman, E., et al., Shear wave elastography quantifies stiffness in ex vivo porcine artery with stiffened arterial region. Ultrasound in medicine \& biology, 2016. 42(10): p. 2423-2435.

12. Ramnarine, K.V., et al., Shear wave elastography imaging of carotid plaques: feasible, reproducible and of clinical potential. Cardiovascular ultrasound, 2014. 12(1): p. 49.

13. Garrard, J.W., et al., Shear wave elastography may be superior to greyscale median for the identification of carotid plaque vulnerability: A comparison with histology. Ultraschall in der MedizinEuropean Journal of Ultrasound, 2015. 36 (04), 386-390.

14. Ramnarine, K.V., et al., Shear wave elastography assessment of carotid plaque stiffness: in vitro reproducibility study. Ultrasound in medicine \& biology, 2014. 40 (1), 200-209

15. Widman, E., et al., Shear wave elastography plaque characterization with mechanical testing validation: a phantom study. Physics in Medicine \& Biology, 2015. 60(8): p. 3151.

\section{Figures}

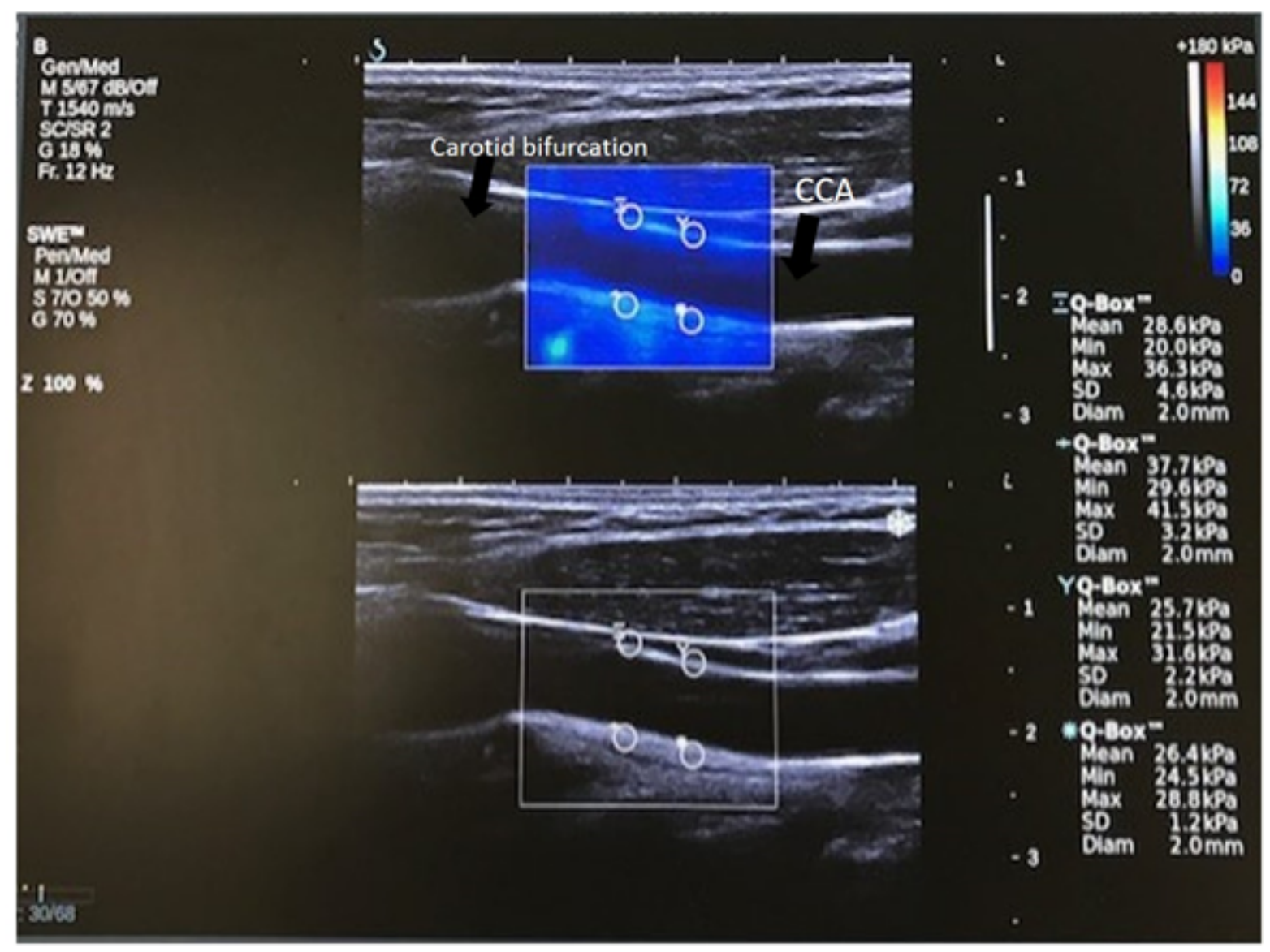

\section{Figure 1}

Example of a single CCA image frame showing placement of 4 Rols for estimation of mean YM. Top image shows SWE image box and bottom B-mode image. 


$$
p=0.32
$$

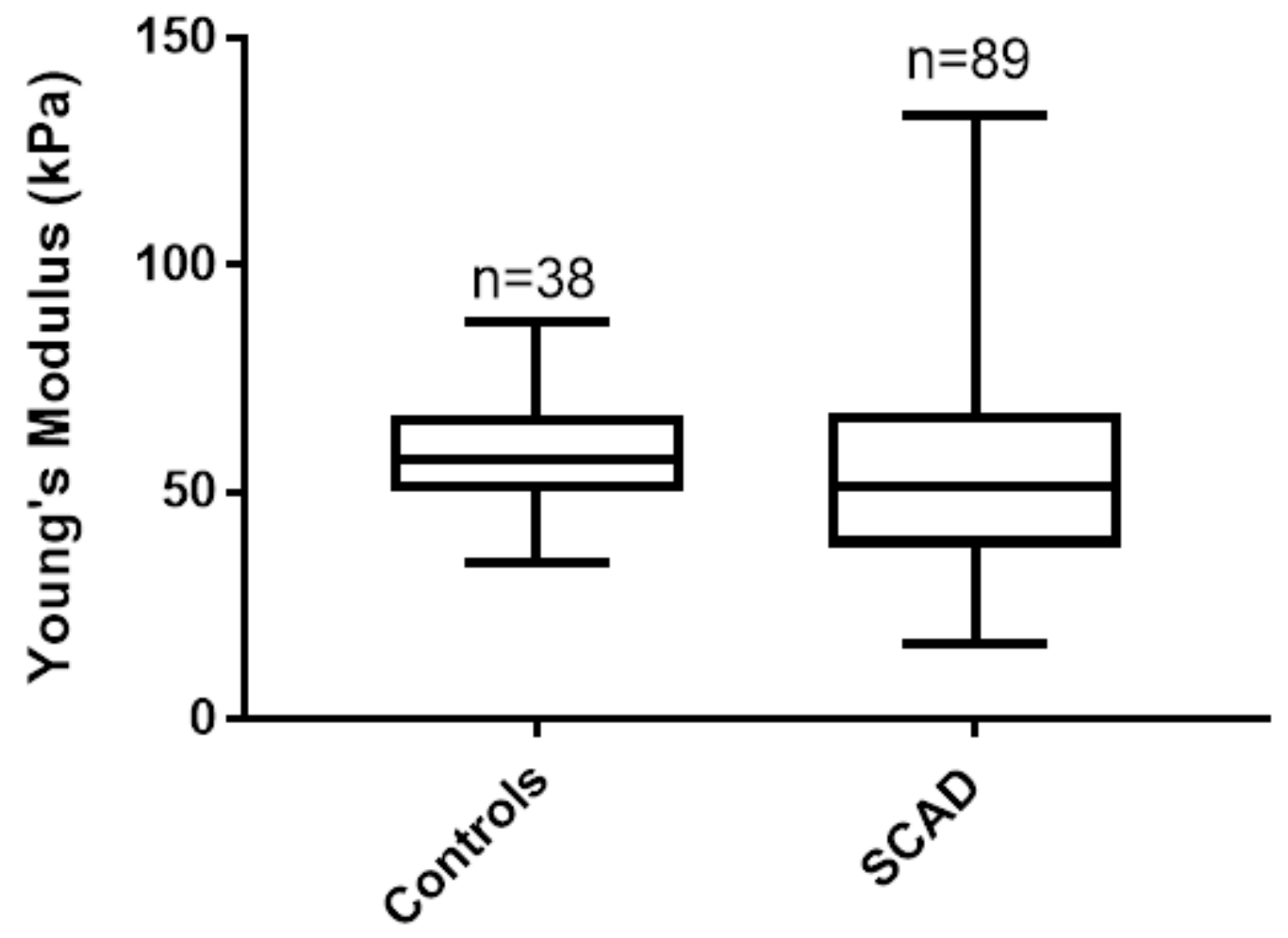

Figure 2

Box and Whisker plot of mean YM estimates in the CCAs of SCAD patients and non-dissection controls. 